\title{
Delay-induced Hopf bifurcation of an SVEIR computer virus model with nonlinear incidence rate
}

\author{
Tao Zhao $^{1 *}$, Zizhen Zhang ${ }^{1}$ and Ranjit Kumar Upadhyay ${ }^{2}$
}

\author{
"Correspondence: \\ zhaotaoaufe@163.com \\ 'School of Management Science \\ and Engineering, Anhui University \\ of Finance and Economics, Bengbu, \\ China \\ Full list of author information is \\ available at the end of the article
}

\begin{abstract}
We are concerned with the Hopf bifurcation of an SVEIR computer virus model with time delay and nonlinear incident rate. First of all, by analyzing the associated characteristic equation we obtain sufficient conditions for its local stability and the existence of a Hopf bifurcation. Directly afterward, by means of the normal form theory and the center manifold theorem we derive explicit formulas that determine the direction of the Hopf bifurcation and the stability of the bifurcated periodic solutions. Finally, we carry out numerical simulations to illustrate and verify the theoretical results.
\end{abstract}

MSC: 34C15; 34C23; 37G15; 37N25

Keywords: Delay; Hopf bifurcation; Nonlinear incidence rate; SVEIR model; Periodic solutions

\section{Introduction}

With the fast development and popularization of computer networks, computer viruses have tremendous influence on our society. To predict the propagation of computer viruses in networks, in recent years many computer virus models have been proposed and investigated such as SIRS models [1-5], SEIS models [6, 7], SEIR models [8-10], SEIQRS models [11-13], SLBS models [14-16] and some other models [17-20].

The overwhelming majority of the computer virus models mentioned assume a bilinear infection rate. However, there are several reasons why bilinear infection rate requires modification [21]. Especially, the propagation of computer viruses can be dramatically affected by the topology of the underlying network, and this may lead to some specific nonlinear infection rates. In addition, the choice of the treatment function is also an important factor for the modeling of computer virus spreading. For example, the treatment rate may be slow due to the lower effectiveness of antivirus, and the treatment rate may increase slowly and attain its peak and finally settles down at its saturation value with the improved and effective antivirus technology [22]. Based on this fact, Upadhyay et al. [22] proposed the following computer virus model with nonlinear incidence rate and saturated

(c) The Author(s) 2018. This article is distributed under the terms of the Creative Commons Attribution 4.0 International License (http://creativecommons.org/licenses/by/4.0/), which permits unrestricted use, distribution, and reproduction in any medium, provided you give appropriate credit to the original author(s) and the source, provide a link to the Creative Commons license, and indicate if changes were made. 
treatment rate:

$$
\left\{\begin{array}{l}
\frac{d S(t)}{d t}=A-\delta_{0} S(t)-\frac{\alpha S(t) I(t)}{S(t)+I(t)+c}+\eta V(t)-\mu S(t), \\
\frac{d E(t)}{d t}=\frac{\alpha S(t) I(t)}{S(t)+I(t)+c}-\left(\delta_{0}+\delta_{1}\right) E(t), \\
\frac{d I(t)}{d t}=\delta_{1} E(t)-\left(\delta_{0}+\delta_{2}+\delta_{3}\right) I(t)-\frac{\beta I(t)}{I(t)+a} \\
\frac{d R(t)}{d t}=\delta_{2} I(t)-\delta_{0} R(t)+\frac{\beta I(t)}{I(t)+a} \\
\frac{d V(t)}{d t}=\mu S(t)-\left(\delta_{0}+\eta\right) V(t)
\end{array}\right.
$$

where $S(t), E(t), I(t), R(t)$, and $V(t)$ denote the numbers of the susceptible computers, the exposed computers, the infectious computers, the recovered computers, and the vaccinated computers at time $t$, respectively, $A$ is the recruitment rate of new computers, $\alpha$ is the contact rate of the susceptible computers, $\eta$ is the rate at which the vaccinated computers lose their immunity and join the susceptible ones, $\beta$ denotes the maximal treatment capacity of a network, $\delta_{0}$ is the natural mortality rate of all the computers, $\delta_{1}$ is the rate at which the exposed computers become the infectious ones, $\delta_{2}$ is the recovery rate of the infectious computers, $\delta_{3}$ is the crashing rate of the infectious computers due to the viruses, $a$ is the half saturation constant for the infectious computers, $c$ is the saturation constant for the susceptible computers, and $\mu$ is the vaccination rate of the susceptible computers. Upadhyay et al. [22] studied the stability of the viral equilibrium of system (1).

It is well known that time delays of one type or another have been incorporated into computer virus models due to latent period [3, 4], temporary immunity period $[5,12]$, or other reasons [9], because time delays may play a complicated role on the models. For example, time delays can cause the loss of stability and can induce Hopf bifurcation and periodic solutions. As stated in [4], the occurrence of a Hopf bifurcation means that the state of computer virus prevalence changes from an equilibrium to a limit cycle, and this phenomenon is unexpected, since the periodic behavior is unpleasant from the viewpoint of epidemiology. Having this idea in mind and considering that the antivirus software may use a period to clean the viruses in the infectious computers, it is worth investigating the Hopf bifurcation of the following system with time delay:

$$
\left\{\begin{array}{l}
\frac{d S(t)}{d t}=A-\delta_{0} S(t)-\frac{\alpha S(t) I(t)}{S(t)+I(t)+c}+\eta V(t)-\mu S(t), \\
\frac{d E(t)}{d t}=\frac{\alpha S(t) I(t)}{S(t)+I(t)+c}-\left(\delta_{0}+\delta_{1}\right) E(t), \\
\frac{d I(t)}{d t}=\delta_{1} E(t)-\left(\delta_{0}+\delta_{3}\right) I(t)-\delta_{2} I(t-\tau)-\frac{\beta I(t-\tau)}{I(t-\tau)+a}, \\
\frac{d R(t)}{d t}=\delta_{2} I(t-\tau)-\delta_{0} R(t)+\frac{\beta I(t-\tau)}{I(t-\tau)+a}, \\
\frac{d V(t)}{d t}=\mu S(t)-\left(\delta_{0}+\eta\right) V(t),
\end{array}\right.
$$

where $\tau$ is the time delay due to the period the antivirus software uses to clean the viruses in the infectious computers.

The organization of the rest of this paper is organized as follows. In Sect. 2, the local stability and existence of a Hopf bifurcation are performed. In Sect. 3, the direction and stability of the Hopf bifurcation are determined. In Sect. 4, the obtained analytical findings are justified through computer simulations. This work is closed by Sect. 5 . 


\section{Stability of the viral equilibrium and existence of Hopf bifurcation}

By direct computation we get that if $\left[\alpha \delta_{1}-\left(\delta_{0}+\delta_{1}\right)\left(\delta_{0}+\delta_{2}+\delta_{3}\right)\right]\left(I_{*}+a\right)>\beta \delta_{1}\left(\delta_{0}+\delta_{1}\right)$, then system (2) has a viral equilibrium $P_{*}\left(S_{*}, E_{*}, I_{*}, R_{*}, V_{*}\right)$, where

$$
\begin{aligned}
S_{*} & =\frac{\left(\delta_{0}+\delta_{1}\right)\left(\delta_{0}+\delta_{2}+\delta_{3}\right)\left(I_{*}+a\right)\left(I_{*}+c\right)+\beta \delta_{1}\left(\delta_{0}+\delta_{1}\right)\left(I_{*}+c\right)}{\left[\alpha \delta_{1}-\left(\delta_{0}+\delta_{1}\right)\left(\delta_{0}+\delta_{2}+\delta_{3}\right)\right]\left(I_{*}+a\right)-\beta \delta_{1}\left(\delta_{0}+\delta_{1}\right)}, \\
E_{*} & =\frac{\left(\delta_{0}+\delta_{2}+\delta_{3}\right) I_{*}}{\delta_{1}}+\frac{\beta I_{*}}{\delta_{1}\left(I_{*}+a\right)}, \\
R_{*} & =\frac{\delta_{2} I_{*}}{\delta_{0}}+\frac{\beta I_{*}}{\delta_{0}\left(I_{*}+a\right)}, \\
V_{*} & =\frac{\mu\left[\left(\delta_{0}+\delta_{1}\right)\left(\delta_{0}+\delta_{2}+\delta_{3}\right)\left(I_{*}+a\right)\left(I_{*}+c\right)+\beta \delta_{1}\left(\delta_{0}+\delta_{1}\right)\left(I_{*}+c\right)\right]}{\left(\delta_{0}+\eta\right)\left\{\left[\alpha \delta_{1}-\left(\delta_{0}+\delta_{1}\right)\left(\delta_{0}+\delta_{2}+\delta_{3}\right)\right]\left(I_{*}+a\right)-\beta \delta_{1}\left(\delta_{0}+\delta_{1}\right)\right\}},
\end{aligned}
$$

and $I_{*}$ is the positive root of the equation

$$
a_{3} I^{3}+a_{2} I^{2}+a_{1} I+a_{0}=0
$$

where

$$
\begin{aligned}
a_{0}= & \delta_{1} B_{6} c-B_{1} c\left(\delta_{0}+\eta\right)+\delta_{1} a c\left[B_{7}-B_{2}\left(\delta_{0}+\eta\right)\right] \\
& +A a \delta_{1}\left(\delta_{0}+\eta\right)\left(B_{3} a-B_{4}\right), \\
a_{1}= & B_{6} a^{2} \delta_{1}-a^{2} \delta_{1}\left(\delta_{0}+\eta\right)\left(B_{1} \delta_{1}+B_{3} B_{5}\right) \\
& +\delta_{1}(a+c)\left(B_{7}-B_{2}\left(\delta_{0}+\eta\right)\right) \\
& +a\left(\delta_{0}+\eta\right)\left(B_{4} B_{5} \delta_{1}-B_{3} \beta\left(\delta_{0}+\delta_{1}\right)\right) \\
& +\left(\delta_{0}+\eta\right)\left(2 A B_{3} a \delta_{1}-A B_{4} \delta_{1}+B_{4} \beta\left(\delta_{0}+\delta_{1}\right)\right), \\
a_{2}= & \delta_{1}\left(2 B_{6} a+B_{7}\right)+\delta_{1}\left(\delta_{0}+\eta\right)\left(A B_{3}+B_{4} B_{5}-B_{2}\right) \\
& -\left(\delta_{0}+\eta\right)\left(B_{3} \beta\left(\delta_{0}+\delta_{1}\right)+\delta_{1}\left(B_{1}+B_{3} B_{5}\right)\right), \\
a_{3}= & \delta_{1} B_{6}-\delta_{1}\left(\delta_{0}+\eta\right)\left(B_{1}+B_{3} B_{5}\right),
\end{aligned}
$$

with

$$
\begin{aligned}
& B_{1}=\left(\delta_{0}+\mu\right)\left(\delta_{0}+\delta_{1}\right)\left(\delta_{0}+\delta_{2}+\delta_{3}\right), \\
& B_{2}=\beta \delta_{1}\left(\delta_{0}+\mu\right)\left(\delta_{0}+\delta_{1}\right), \\
& B_{3}=\alpha \delta_{1}-\left(\delta_{0}+\delta_{1}\right)\left(\delta_{0}+\delta_{2}+\delta_{3}\right), \\
& B_{4}=\beta \delta_{1}\left(\delta_{0}+\delta_{1}\right), \\
& B_{5}=\frac{\left(\delta_{0}+\delta_{1}\right)\left(\delta_{0}+\delta_{2}+\delta_{3}\right)}{\delta_{1}}, \\
& B_{6}=\eta \mu\left(\delta_{0}+\delta_{1}\right)\left(\delta_{0}+\delta_{2}+\delta_{3}\right), \\
& B_{7}=\eta \mu \beta \delta_{1}\left(\delta_{0}+\delta_{1}\right) .
\end{aligned}
$$

For Eq. (3), we have the following results. 


\section{Lemma 1 If $a_{3}=0$, then}

(1) if $a_{2}=0$ and $a_{0} / a_{1}<0$, then there exists a unique positive root $I_{*}=-a_{0} / a_{1}$ of Eq. (3);

(2) When $\Delta>0$, if $a_{2} / a_{1}<0$ and $a_{0} / a_{2}>0$, then there exist two positive roots $I_{*}^{(1)}=I_{*}^{+}$ and $I_{*}^{(2)}=I_{*}^{-}$; if $a_{0} / a_{2}<0$, then there is a unique positive root $I_{*}=I_{*}^{+}$with $a_{1}>0$ or $I_{*}=I_{*}^{-}$with $a_{2}<0$; if $a_{0}=0$ and $a_{1} / a_{2}<0$, then there is a unique positive root $I_{*}=-a_{1} / a_{2}$

(3) if $\Delta=0$ and $a_{1} / a_{2}<0$, then there is a unique positive root $I_{*}=-a_{1} /\left(2 a_{2}\right)$. Here $\Delta=a_{1}^{2}-4 a_{2} a_{0}, I_{*}^{+}=\left(-a_{1}+\sqrt{\Delta}\right) /\left(2 a_{2}\right)$, and $I_{*}^{-}=-\left(a_{1}+\sqrt{\Delta}\right) /\left(2 a_{2}\right)$.

Lemma 2 For $a_{3} \neq 0$, let $l_{2}=a_{2} / a_{3}, l_{1}=a_{1} / a_{3}$, and $l_{0}=a_{0} / a_{3}$. Then:

(1) if $l_{0}<0$, then $E q$. (3) has at least one positive root;

(2) if $l_{0} \geq 0$ and $l_{2}^{2}-3 l_{1} \leq 0$, then $E q$. (3) has no positive root;

(3) if $l_{0} \geq 0$ and $l_{2}^{2}-3 l_{1}>0$, then Eq. (3) has a positive root if and only if $\frac{-l_{2}+\sqrt{l_{2}^{2}-3 l_{1}}}{3}>0$ and $h\left(\frac{-l_{2}+\sqrt{l_{2}^{2}-3 l_{1}}}{3}\right) \leq 0$, where $h(I)=I^{3}+l_{2} I^{2}+l_{1} I+l_{0}$.

Then, we can obtain the linearization of system (2). Let $u_{1}(t)=S(t)-S_{*}, u_{2}(t)=E(t)-E_{*}$, $u_{3}(t)=I(t)-I_{*}, u_{4}(t)=R(t)-R_{*}, u_{5}(t)=V(t)-V_{*}$. We can rewrite system (2) as follows:

$$
\left\{\begin{array}{l}
\dot{u}_{1}(t)=a_{11} u_{1}(t)+a_{13} u_{3}(t)+a_{15} u_{5}(t)+\sum_{i+j \geq 2} \frac{1}{i ! j} f_{i j}^{(1)} u_{1}^{i}(t) u_{3}^{j}(t) \\
\dot{u}_{2}(t)=a_{21} u_{1}(t)+a_{22} u_{2}(t)+a_{23} u_{3}(t)+\sum_{i+j \geq 2} \frac{1}{i ! j} f_{i j}^{(2)} u_{1}^{i}(t) u_{3}^{j}(t) \\
\dot{u}_{3}(t)=a_{32} u_{2}(t)+a_{33} u_{3}(t)+b_{33} u_{3}(t-\tau)+\sum_{i \geq 2} \frac{1}{i} f_{i}^{(3)} u_{3}^{i}(t-\tau) \\
\dot{u}_{4}(t)=a_{44} u_{4}(t)+b_{43} u_{3}(t-\tau)+\sum_{i \geq 2} \frac{1}{i} f_{i}^{(4)} u_{3}^{i}(t-\tau) \\
\dot{u}_{5}(t)=a_{51} u_{1}(t)+a_{55} u_{5}(t)
\end{array}\right.
$$

where

$$
\begin{aligned}
& a_{11}=-\left[\delta_{0}+\mu+\frac{\alpha I_{*}\left(I_{*}+c\right)}{\left(S_{*}+I_{*}+c\right)^{2}}\right], \quad a_{13}=-\frac{\alpha S_{*}\left(S_{*}+c\right)}{\left(S_{*}+I_{*}+c\right)^{2}}, \quad a_{15}=\eta, \\
& a_{21}=\frac{\alpha I_{*}\left(I_{*}+c\right)}{\left(S_{*}+I_{*}+c\right)^{2}}, \quad a_{22}=-\left(\delta_{0}+\delta_{1}\right), \quad a_{23}=\frac{\alpha S_{*}\left(S_{*}+c\right)}{\left(S_{*}+I_{*}+c\right)^{2}}, \\
& a_{32}=\delta_{1}, \quad a_{33}=-\left(\delta_{0}+\delta_{3}\right), \quad b_{33}=-\left[\delta_{2}+\frac{a \beta}{\left(I_{*}+a\right)^{2}}\right], \\
& a_{44}=-\delta_{0}, \quad a_{51}=\mu,\left(\delta_{0}+\eta\right), \\
& b_{43}=\left[\delta_{2}+\frac{a \beta}{\left(I_{*}+a\right)^{2}}\right], \\
& f_{i j}^{(k)}=\frac{\partial^{i+j} f^{(k)}\left(S_{*}, E_{*}, I_{*}, R_{*}, V_{*}\right)}{\partial u_{1}^{i}(t) \partial u_{3}^{j}(t)}, \\
& f_{i}^{(k)}=\frac{\partial^{i} f^{(k)}\left(S_{*}, E_{*}, I_{*}, R_{*}, V_{*}\right)}{\partial u_{3}^{i}(t-\tau)}, \\
& f^{(1)}=A-\delta_{0} u_{1}(t)-\frac{\alpha u_{1}(t) u_{3}(t)}{u_{1}(t)+u_{3}(t)+c}-\mu S(t), \\
& f^{(2)}=\frac{\alpha u_{1}(t) u_{3}(t)}{u_{1}(t)+u_{3}(t)+c}-\left(\delta_{0}+\delta_{1}\right) u_{2}(t),
\end{aligned}
$$




$$
\begin{aligned}
& f^{(3)}=\delta_{1} u_{2}(t)-\left(\delta_{0}+\delta_{3}\right) u_{3}(t)-\delta_{2} u_{3}(t-\tau)-\frac{\beta u_{3}(t-\tau)}{u_{3}(t-\tau)+a}, \\
& f^{(4)}=\delta_{2} u_{3}(t-\tau)-\delta_{0} u_{4}(t)+\frac{\beta u_{3}(t-\tau)}{u_{3}(t-\tau)+a}
\end{aligned}
$$

Then we obtain the linearized system of system (4)

$$
\left\{\begin{array}{l}
\dot{u}_{1}(t)=a_{11} u_{1}(t)+a_{13} u_{3}(t)+a_{15} u_{5}(t), \\
\dot{u}_{2}(t)=a_{21} u_{1}(t)+a_{22} u_{2}(t)+a_{23} u_{3}(t), \\
\dot{u}_{3}(t)=a_{32} u_{2}(t)+a_{33} u_{3}(t)+b_{33} u_{3}(t-\tau), \\
\dot{u}_{4}(t)=a_{44} u_{4}(t)+b_{43} u_{3}(t-\tau), \\
\dot{u}_{5}(t)=a_{51} u_{1}(t)+a_{55} u_{5}(t) .
\end{array}\right.
$$

The characteristic equation is

$$
\begin{aligned}
P(\lambda) & =\lambda^{5}+p_{4} \lambda^{4}+p_{3} \lambda^{3}+p_{2} \lambda^{2}+p_{1} \lambda+p_{0}+\left(q_{4} \lambda^{4}+q_{3} \lambda^{3}+q_{2} \lambda^{2}+q_{1} \lambda+q_{0}\right) e^{-\lambda \tau} \\
& =0
\end{aligned}
$$

where

$$
\begin{aligned}
p_{0}= & a_{44}\left(a_{22} a_{33}-a_{23} a_{32}\right)\left(a_{15} a_{51}-a_{11} a_{55}\right)-a_{13} a_{21} a_{32} a_{44} a_{55}, \\
p_{1}= & a_{55}\left(a_{11} a_{22}\left(a_{33}+a_{44}\right)+a_{33} a_{44}\left(a_{11}+a_{22}\right)\right)+a_{11} a_{22} a_{33} a_{44} \\
& -a_{23} a_{32}\left(a_{11} a_{44}+a_{11} a_{55}+a_{44} a_{55}\right)+a_{15} a_{23} a_{32} a_{51} \\
& -a_{15} a_{51}\left(a_{22} a_{33}+a_{22} a_{44}+a_{33} a_{44}\right)+a_{13} a_{21} a_{32}\left(a_{44}+a_{55}\right), \\
p_{2}= & a_{23} a_{32}\left(a_{11}+a_{44}+a_{55}\right)+a_{15} a_{51}\left(a_{22}+a_{33}+a_{44}\right)-a_{13} a_{21} a_{32} \\
& -\left(a_{11} a_{22}\left(a_{33}+a_{44}\right)+a_{33} a_{44}\left(a_{11}+a_{22}\right)\right) \\
& -a_{55}\left(a_{11} a_{22}+a_{33} a_{44}+\left(a_{11}+a_{22}\right)\left(a_{33}+a_{44}\right)\right), \\
p_{3}= & a_{11} a_{22}+a_{33} a_{44}+\left(a_{11}+a_{22}\right)\left(a_{33}+a_{44}\right)-a_{23} a_{32}-a_{15} a_{51} \\
& +a_{55}\left(a_{11}+a_{22}+a_{33}+a_{44}\right), \\
p_{4}= & -\left(a_{11}+a_{22}+a_{33}+a_{44}+a_{55}\right), \quad q_{0}=a_{22} a_{44} b_{33}\left(a_{15} a_{51}-a_{11} a_{55}\right), \\
q_{1}= & a_{11} a_{22} b_{33}\left(a_{44}+a_{55}\right)+a_{44} a_{55} b_{33}\left(a_{11}+a_{22}\right)-a_{15} a_{51} b_{33}\left(a_{22}+a_{44}\right), \\
q_{2}= & a_{15} a_{51} b_{33}-b_{33}\left(a_{11} a_{22}+a_{44} a_{55}+\left(a_{11}+a_{22}\right)\left(a_{44}+a_{55}\right)\right), \\
q_{3}= & b_{33}\left(a_{11}+a_{22}+a_{44}+a_{55}\right), \quad q_{4}=-b_{33} .
\end{aligned}
$$

When $\tau=0$, Eq. (3) reduces to

$$
\lambda^{5}+p_{04} \lambda^{4}+p_{03} \lambda^{3}+p_{02} \lambda^{2}+p_{01} \lambda+p_{00}=0
$$

with

$$
\begin{array}{ll}
p_{00}=p_{0}+q_{0}, & p_{01}=p_{1}+q_{1}, \quad p_{02}=p_{2}+q_{2}, \\
p_{03}=p_{3}+q_{3}, & p_{04}=p_{4}+q_{4} .
\end{array}
$$


Obviously,

$$
p_{04}=\mu+5 \delta_{0}+\delta_{1}+\delta_{2}+\delta_{3}+\frac{\alpha I_{*}\left(I_{*}+c\right)}{\left(S_{*}+I_{*}+c\right)^{2}}+\frac{a \beta}{\left(I_{*}+a\right)^{2}}>0 .
$$

An application of the Routh-Hurwitz criterion gives $\operatorname{Re}(\lambda)<0$ if and only if condition $\left(H_{1}\right)$ is satisfied, that is, if the following inequalities hold:

$$
\begin{aligned}
\operatorname{det}_{2} & =\left|\begin{array}{cc}
p_{04} & 1 \\
p_{02} & p_{03}
\end{array}\right|>0, \\
\operatorname{det}_{3} & =\left|\begin{array}{ccc}
p_{04} & 1 & 0 \\
p_{02} & p_{03} & p_{04} \\
0 & p_{01} & p_{02}
\end{array}\right|>0 \\
\operatorname{det}_{4} & =\left|\begin{array}{cccc}
p_{04} & 1 & 0 & 0 \\
p_{02} & p_{03} & p_{04} & 1 \\
p_{00} & p_{01} & p_{02} & p_{03} \\
0 & 0 & p_{00} & p_{01}
\end{array}\right|>0 \\
\operatorname{det}_{5} & =\left|\begin{array}{ccccc}
p_{04} & 1 & 0 & 0 & 0 \\
p_{02} & p_{03} & p_{04} & 1 & 0 \\
p_{00} & p_{01} & p_{02} & p_{03} & p_{04} \\
0 & 0 & p_{00} & p_{01} & p_{02} \\
0 & 0 & 0 & 0 & p_{00}
\end{array}\right|>0 .
\end{aligned}
$$

For $\tau>0$, we assume that $\lambda=i \omega(\omega>0)$ is a root of Eq. (6). Then

$$
\left\{\begin{array}{l}
\left(q_{1} \omega-q_{3} \omega^{3}\right) \sin \tau \omega+\left(q_{4} \omega^{4}-q_{2} \omega^{2}+q_{0}\right) \cos \tau \omega=p_{2} \omega^{2}-p_{4} \omega^{4}-p_{0} \\
\left(q_{1} \omega-q_{3} \omega^{3}\right) \cos \tau \omega-\left(q_{4} \omega^{4}-q_{2} \omega^{2}+q_{0}\right) \sin \tau \omega=p_{3} \omega^{3}-\omega^{5}-p_{1} \omega
\end{array}\right.
$$

Thus

$$
\omega^{10}+e_{4} \omega^{8}+e_{3} \omega^{6}+e_{2} \omega^{4}+e_{1} \omega^{2}+e_{0}=0
$$

where

$$
\begin{aligned}
& e_{0}=p_{0}^{2}-q_{0}^{2}, \quad e_{1}=p_{1}^{2}-2 p_{0} p_{2}+2 q_{0} q_{2}-q_{1}^{2}, \\
& e_{2}=p_{2}^{2}-2 p_{1} p_{3}+2 p_{0} p_{4}-q_{2}^{2}-2 q_{1} q_{3}, \\
& e_{3}=p_{3}^{2}+2 p_{1}-2 p_{2} p_{4}+2 q_{2} q_{4}-q_{3}^{2}, \\
& e_{4}=p_{4}^{2}-2 p_{3}-q_{4}^{2} .
\end{aligned}
$$

Let $v=\omega^{2}$. Then Eq. (12) becomes

$$
v^{5}+e_{4} v^{4}+e_{3} v^{3}+e_{2} v^{2}+e_{1} v+e_{0}=0
$$


Based on the discussion about the distribution of the roots of Eq. (13) in [23] and considering that all the values of parameters in system (2) are given, we can obtain all the roots of Eq. (13). Thus we make the following assumption:

$\left(H_{2}\right)$ Equation (13) has at least one positive root $v_{0}$.

If condition $\left(H_{2}\right)$ holds, then there exists $v_{0}>0$ such that Eq. (6) has a pair of purely imaginary roots $\pm i \omega_{0}= \pm i \sqrt{v_{0}}$. For $\omega_{0}$, we have

$$
\tau_{0}=\frac{1}{\omega_{0}} \times\left\{\frac{g_{1}\left(\omega_{0}\right)}{g_{2}\left(\omega_{0}\right)}\right\}
$$

where

$$
\begin{aligned}
g_{1}\left(\omega_{0}\right)= & \left(q_{3}-p_{4} q_{4}\right) \omega_{0}^{8}+\left(p_{3} q_{3}-q_{1}+p_{2} q_{4}+p_{4} q 2\right) \omega_{0}^{6} \\
& +\left(p_{1} q_{3}+p_{3} q_{1}-p_{0} q_{4}-p_{2} q_{2}-p_{4} q_{0}\right) \omega_{0}^{4} \\
& +\left(p_{0} q_{2}+p_{2} q_{0}-p_{1} q_{1}\right) \omega_{0}^{2}-p_{0} q_{0}, \\
g_{2}\left(\omega_{0}\right)= & q_{4}^{2} \omega_{0}^{8}+\left(q_{3}^{2}-2 q_{2} q_{4}\right) \omega_{0}^{6}+\left(q_{2}^{2}+2 q_{0} q_{4}+2 q_{1} q_{3}\right) \omega_{0}^{4} \\
& +\left(q_{1}^{2}-2 q_{0} q_{2}\right) \omega_{0}^{2}+q_{0}^{2} .
\end{aligned}
$$

Next, differentiating Eq. (6) with respect to $\tau$, we obtain

$$
\begin{aligned}
{\left[\frac{d \lambda}{d \tau}\right]^{-1}=} & -\frac{5 \lambda^{4}+4 p_{4} \lambda^{3}+3 p_{3} \lambda^{2}+2 p_{2} \lambda+p_{1}}{\lambda\left(\lambda^{5}+p_{4} \lambda^{4}+p_{3} \lambda^{3}+p_{2} \lambda^{2}+p_{1} \lambda+p_{0}\right)} \\
& +\frac{4 q_{4} \lambda^{3}+3 q_{3} \lambda^{2}+2 q_{2} \lambda+q_{1}}{\lambda\left(q_{4} \lambda^{4}+q_{3} \lambda^{3}+q_{2} \lambda^{2}+q_{1} \lambda+q_{0}\right)}-\frac{\tau}{\lambda} .
\end{aligned}
$$

Further, we have

$$
\operatorname{Re}\left[\frac{d \lambda}{d \tau}\right]_{\tau=\tau_{0}}^{-1}=\frac{f^{\prime}\left(v_{0}\right)}{\left(q_{1} \omega_{0}-q_{3} \omega_{0}^{3}\right)^{2}+\left(q_{4} \omega_{0}^{4}-q_{2} \omega_{0}^{2}+q_{0}\right)^{2}},
$$

where $v_{0}=\omega_{0}^{2}$ and $f(v)=v^{5}+e_{4} v^{4}+e_{3} v^{3}+e_{2} v^{2}+e_{1} v+e_{0}$.

Therefore, if condition $\left(H_{3}\right): f^{\prime}\left(v_{0}\right) \neq 0$ holds, then $\operatorname{Re}\left[\frac{d \lambda}{d \tau}\right]_{\tau=\tau_{0}} \neq 0$. Based on the previous discussion and the Hopf bifurcation theorem in [24], we have the following:

Theorem 1 Suppose that the conditions $\left(H_{1}\right),\left(H_{2}\right)$, and $\left(H_{3}\right)$ hold for system (2). The viral equilibrium $P_{*}\left(S_{*}, E_{*}, I_{*}, R_{*}, V_{*}\right)$ is locally asymptotically stable when $\tau \in\left[0, \tau_{0}\right)$; a Hopf bifurcation occurs at the viral equilibrium $P_{*}\left(S_{*}, E_{*}, I_{*}, R_{*}, V_{*}\right)$ when $\tau=\tau_{0}$, and a family of periodic solutions bifurcate from the viral equilibrium $P_{*}\left(S_{*}, E_{*}, I_{*}, R_{*}, V_{*}\right)$ near $\tau=\tau_{0}$.

\section{Direction and stability of the Hopf bifurcation}

Let $u_{1}(t)=S(t)-S_{*}, u_{2}(t)=E(t)-E_{*}, u_{3}(t)=I(t)-I_{*}, u_{4}(t)=R(t)-R_{*}, u_{5}(t)=V(t)-V_{*}$. Rescale the time delay by $t \rightarrow(t / \tau)$. Let $\tau=\tau_{0}+\varrho, \varrho \in R$. Then the Hopf bifurcation occurs at $\varrho=0$. Thus system (2) can be transformed into

$$
\dot{u}(t)=L_{\varrho} u_{t}+F\left(\varrho, u_{t}\right)
$$


where $u_{t}=\left(u_{1}(t), u_{2}(t), u_{3}(t), u_{4}(t), u_{5}(t)\right)^{T}=(S, E, I, R, V)^{T} \in R^{5}, u_{t}(\theta)=u(t+\theta) \in C=$ $C\left([-1,0], R^{5}\right)$, and $L_{\varrho}: C \rightarrow R^{5}$ and $F\left(\varrho, u_{t}\right) \rightarrow R^{5}$ are given by

$$
\begin{aligned}
& L_{\varrho} \phi=\left(\tau_{0}+\varrho\right)\left(M_{\max } \phi(0)+N_{\max } \phi(-1)\right), \\
& F(\varrho, \phi)=\left(F_{1}, F_{2}, F_{3}, F_{4}, 0\right)
\end{aligned}
$$

with

$$
M_{\max }=\left(\begin{array}{ccccc}
a_{11} & 0 & a_{13} & 0 & a_{15} \\
a_{21} & a_{22} & a_{23} & 0 & 0 \\
0 & a_{32} & a_{33} & 0 & 0 \\
0 & 0 & 0 & a_{44} & 0 \\
a_{51} & 0 & 0 & 0 & a_{55}
\end{array}\right), \quad N_{\max }=\left(\begin{array}{ccccc}
0 & 0 & 0 & 0 & 0 \\
0 & 0 & 0 & 0 & 0 \\
0 & 0 & b_{33} & 0 & 0 \\
0 & 0 & b_{43} & 0 & 0 \\
0 & 0 & 0 & 0 & 0
\end{array}\right),
$$

and

$$
\begin{aligned}
& F_{1}=a_{16} \phi_{1}^{2}(0)+a_{17} \phi_{3}^{2}(0)+a_{18} \phi_{1}(0) \phi_{3}(0)+a_{19} \phi_{1}^{2}(0) \phi_{3}(0) \\
& +a_{110} \phi_{1}(0) \phi_{3}^{2}(0)+a_{111} \phi_{1}^{3}(0)+a_{112} \phi_{3}^{3}(0)+\cdots, \\
& F_{2}=a_{24} \phi_{1}^{2}(0)+a_{25} \phi_{3}^{2}(0)+a_{26} \phi_{1}(0) \phi_{3}(0)+a_{27} \phi_{1}^{2}(0) \phi_{3}(0) \\
& +a_{28} \phi_{1}(0) \phi_{3}^{2}(0)+a_{29} \phi_{1}^{3}(0)+a_{210} \phi_{3}^{3}(0)+\cdots, \\
& F_{3}=a_{34} \phi_{3}^{2}(-1)+a_{35} \phi_{3}^{3}(-1)+\cdots, \\
& F_{4}=a_{45} \phi_{3}^{2}(-1)+a_{46} \phi_{3}^{3}(-1)+\cdots, \\
& a_{16}=\frac{\alpha I_{*}\left(I_{*}+c\right)}{\left(S_{*}+I_{*}+c\right)^{3}}, \quad a_{17}=\frac{\alpha S_{*}\left(S_{*}+c\right)}{\left(S_{*}+I_{*}+c\right)^{3}}, \\
& a_{18}=-\frac{2 \alpha S_{*} I_{*}+c \alpha\left(S_{*}+I_{*}+c\right)}{\left(S_{*}+I_{*}+c\right)^{3}}, \\
& a_{19}=\frac{2 \alpha I_{*}\left(2 S_{*}-I_{*}\right)+2 c \alpha\left(S_{*}+c\right)}{\left(S_{*}+I_{*}+c\right)^{4}}, \\
& a_{110}=\frac{2 \alpha S_{*}\left(2 I_{*}-S_{*}\right)+2 c \alpha\left(I_{*}+c\right)}{\left(S_{*}+I_{*}+c\right)^{4}}, \\
& a_{111}=-\frac{\alpha I_{*}\left(I_{*}+c\right)}{\left(S_{*}+I_{*}+c\right)^{4}}, \quad a_{112}=-\frac{\alpha S_{*}\left(S_{*}+c\right)}{\left(S_{*}+I_{*}+c\right)^{4}}, \\
& a_{24}=-\frac{\alpha I_{*}\left(I_{*}+c\right)}{\left(S_{*}+I_{*}+c\right)^{3}}, \quad a_{25}=-\frac{\alpha S_{*}\left(S_{*}+c\right)}{\left(S_{*}+I_{*}+c\right)^{3}}, \\
& a_{26}=\frac{2 \alpha S_{*} I_{*}+c \alpha\left(S_{*}+I_{*}+c\right)}{\left(S_{*}+I_{*}+c\right)^{3}}, \\
& a_{27}=-\frac{2 \alpha I_{*}\left(2 S_{*}-I_{*}\right)+2 c \alpha\left(S_{*}+c\right)}{\left(S_{*}+I_{*}+c\right)^{4}}, \\
& a_{28}=-\frac{2 \alpha S_{*}\left(2 I_{*}-S_{*}\right)+2 c \alpha\left(I_{*}+c\right)}{\left(S_{*}+I_{*}+c\right)^{4}}, \\
& a_{29}=\frac{\alpha I_{*}\left(I_{*}+c\right)}{\left(S_{*}+I_{*}+c\right)^{4}}, \quad a_{210}=\frac{\alpha S_{*}\left(S_{*}+c\right)}{\left(S_{*}+I_{*}+c\right)^{4}}, \\
& a_{34}=\frac{a \beta}{\left(I_{*}+a\right)^{3}}, \quad a_{35}=-\frac{a \beta}{\left(I_{*}+a\right)^{4}}, \quad a_{45}=-\frac{a \beta}{\left(I_{*}+a\right)^{3}}, \quad a_{46}=\frac{a \beta}{\left(I_{*}+a\right)^{4}} .
\end{aligned}
$$


According to the Riesz representation theorem, there is a matrix $\eta(\theta, \varrho)$ in $\theta \in[-1,0]$ such that

$$
L_{\mu} \phi=\int_{-1}^{0} d \eta(\theta, \varrho) \phi(\theta)
$$

for $\phi \in C$. In fact, we choose

$$
\eta(\theta, \varrho)=\left(\tau_{0}+\varrho\right)\left(M_{\max } \delta(\theta)+N_{\max } \delta(\theta+1)\right),
$$

where $\delta(\theta)$ is the Dirac delta function.

For $\phi \in C\left([-1,0], R^{5}\right)$, define

$$
A(\varrho) \phi=\left\{\begin{array}{l}
\frac{d \phi(\theta)}{d \theta}, \quad-1 \leq \theta<0, \\
\int_{-1}^{0} d \eta(\theta, \varrho) \phi(\theta), \quad \theta=0,
\end{array}\right.
$$

and

$$
R(\varrho) \phi=\left\{\begin{array}{l}
0, \quad-1 \leq \theta<0 \\
F(\varrho, \phi), \quad \theta=0 .
\end{array}\right.
$$

Then system (14) becomes

$$
\dot{u}(t)=A(\varrho) u_{t}+R(\varrho) u_{t}
$$

For $\varphi \in C^{1}\left([0,1],\left(R^{5}\right)^{*}\right)$, the adjoint operator $A^{*}$ of $A(0)$ can be defined as

$$
A^{*}(\varphi)= \begin{cases}-\frac{d \varphi(s)}{d s}, & 0<s \leq 1 \\ \int_{-1}^{0} d \eta^{T}(s, 0) \varphi(-s), & s=0\end{cases}
$$

Next, we define the bilinear inner form for $A$ and $A^{*}$ :

$$
\langle\varphi(s), \phi(\theta)\rangle=\bar{\varphi}(0) \phi(0)-\int_{\theta=-1}^{0} \int_{\xi=0}^{\theta} \bar{\varphi}(\xi-\theta) d \eta(\theta) \phi(\xi) d \xi
$$

where $\eta(\theta)=\eta(\theta, 0)$.

Let $\rho(\theta)=\left(1, \rho_{2}, \rho_{3}, \rho_{4}, \rho_{5}\right)^{T} e^{i \tau_{0} \omega_{0} \theta}$ and $\rho^{*}(s)=\left(1, \rho_{2}^{*}, \rho_{3}^{*}, \rho_{4}^{*}, \rho_{5}^{*}\right)^{T} e^{i \tau_{0} \omega_{0} s}$ be the eigenvectors for $A(0)$ and $A^{*}(0)$ corresponding to $+i \tau_{0} \omega_{0}$ and $-i \tau_{0} \omega_{0}$, respectively. Then, we have

$$
\begin{aligned}
& \rho_{2}=\frac{a_{21}+a_{23} \rho_{3}}{i \omega_{0}-a_{22}}, \\
& \rho_{3}=\frac{i \omega_{0}-a_{11}}{a_{13}}-\frac{a_{15} a_{51}}{a_{13}\left(i \omega_{0}-a_{55}\right)}, \\
& \rho_{4}=\frac{b_{43} e^{-i \tau_{0} \omega_{0}} \rho_{3}}{i \omega_{0}-a_{44}}, \quad \rho_{5}=\frac{a_{51}}{i \omega_{0}-a_{55}}, \\
& \rho_{2}^{*}=\frac{a_{15} a_{51}}{a_{21}\left(i \omega_{0}+a_{55}\right)}-\frac{i \omega_{0}+a_{11}}{a_{21}},
\end{aligned}
$$




$$
\begin{aligned}
& \rho_{3}^{*}=-\frac{\left(i \omega_{0}+a_{22}\right) \rho_{2}}{a_{32}}, \quad \rho_{5}^{*}=-\frac{a_{15}}{i \omega_{0}+a_{55}}, \\
& \rho_{4}^{*}=-\frac{\left(i \omega_{0}+a_{33}+b_{33} e^{i \tau_{0} \omega_{0}}\right) \rho_{3}^{*}-a_{23} \rho_{2}^{*}+a_{13}}{b_{43} e^{i \tau_{0} \omega_{0}}} .
\end{aligned}
$$

From Eq. (17) we get

$$
\bar{D}=\left[1+\rho_{2} \bar{\rho}_{2}^{*}+\rho_{3} \bar{\rho}_{3}^{*}+\rho_{4} \bar{\rho}_{4}^{*}+\rho_{5} \bar{\rho}_{5}^{*}+\tau_{0} e^{-i \tau_{0} \omega_{0}} \rho_{3}\left(b_{33} \bar{\rho}_{3}^{*}+b_{43} \bar{\rho}_{4}^{*}\right)\right]^{-1},
$$

so that $\left\langle\rho^{*}, \rho\right\rangle=1$ and $\left\langle\rho^{*}, \bar{\rho}\right\rangle=0$.

Next, based on the algorithms in [24] and a computation similar to that in [25-27], we obtain

$$
\begin{aligned}
g_{20}= & 2 \tau_{0} \bar{D}\left[a_{16}+a_{17} \rho_{3}^{2}+a_{18} \rho_{3}+\bar{\rho}_{2}^{*}\left(a_{24}+a_{25} \rho_{3}^{2}+a_{26} \rho_{3}\right)\right. \\
& \left.+\left(a_{34} \bar{\rho}_{3}^{*}+a_{45} \bar{\rho}_{4}^{*}\right) \rho_{3}^{2} e^{-2 i \tau_{0} \omega_{0}}\right], \\
g_{11}= & \tau_{0} \bar{D}\left[2 a_{16}+2 a_{17} \rho_{3} \bar{\rho}_{3}+2 a_{18} \operatorname{Re}\left\{\rho_{3}\right\}+\bar{\rho}_{2}^{*}\left(2 a_{24}+2 a_{25} \rho_{3} \bar{\rho}_{3}+2 a_{26} \operatorname{Re}\left\{\rho_{3}\right\}\right)\right. \\
& \left.+2\left(a_{34} \bar{\rho}_{3}^{*}+a_{45} \bar{\rho}_{4}^{*}\right) \rho_{3} \bar{\rho}_{3}\right], \\
g_{02}= & 2 \tau_{0} \bar{D}\left[a_{16}+a_{17} \bar{\rho}_{3}^{2}+a_{18} \bar{\rho}_{3}+\bar{\rho}_{2}^{*}\left(a_{24}+a_{25} \bar{\rho}_{3}^{2}+a_{26} \bar{\rho}_{3}\right)\right. \\
& \left.+\left(a_{34} \bar{\rho}_{3}^{*}+a_{45} \bar{\rho}_{4}^{*}\right) \bar{\rho}_{3}^{2} e^{2 i \tau_{0} \omega_{0}}\right], \\
g_{21}= & 2 \tau_{0} \bar{D}\left[a_{16}\left(2 W_{11}^{(1)}(0)+W_{20}^{(1)}(0)\right)+a_{17}\left(2 W_{11}^{(3)}(0) \rho_{3}+W_{20}^{(3)}(0) \bar{\rho}_{3}\right)\right. \\
& +a_{18}\left(W_{11}^{(1)}(0) \rho_{3}+\frac{1}{2} W_{20}^{(1)}(0) \bar{\rho}_{3}+W_{11}^{(3)}(0)+\frac{1}{2} W_{20}^{(3)}(0)\right) \\
& +a_{19}\left(\bar{\rho}_{3}+2 \rho_{3}\right)+a_{110}\left(\rho_{3}^{2}+2 \rho_{3} \bar{\rho}_{3}\right)+3 a_{111}+3 a_{112} \rho_{3}^{2} \bar{\rho}_{3} \\
& +\bar{\rho}_{2}^{*}\left(a_{24}\left(2 W_{11}^{(1)}(0)+W_{20}^{(1)}(0)\right)+a_{25}\left(2 W_{11}^{(3)}(0) \rho_{3}+W_{20}^{(3)}(0) \bar{\rho}_{3}\right)\right. \\
& \left.+\bar{\rho}_{4}^{*}\left(a_{45}\left(2 W_{11}^{(3)}(-1) \rho_{3} e^{-i \tau_{0} \omega_{0}}+W_{20}^{(3)}(-1) \bar{\rho}_{3} e^{i \tau_{0} \omega_{0}}\right)+3 a_{46} \rho_{3}^{2} \bar{\rho}_{3} e^{-i \tau_{0} \omega_{0}}\right)\right] \\
& +a_{26}\left(W_{11}^{(1)}(0) \rho_{3}+\frac{1}{2} W_{20}^{(1)}(0) \bar{\rho}_{3}+W_{11}^{(3)}(0)+\frac{1}{2} W_{20}^{(3)}(0)\right) \\
& \left.+a_{27}\left(\bar{\rho}_{3}+2 \rho_{3}\right)+a_{28}\left(\rho_{3}^{2}+2 \rho_{3} \bar{\rho}_{3}\right)+3 a_{29}+3 a_{210} \rho_{3}^{2} \bar{\rho}_{3}\right) \\
& \left.\left.=1) \rho_{3} e^{-i \tau_{0} \omega_{0}}+W_{20}^{(3)}(-1) \bar{\rho}_{3} e^{i \tau_{0} \omega_{0}}\right)+3 a_{35} \rho_{3}^{2} \bar{\rho}_{3} e^{-i \tau_{0} \omega_{0}}\right) \\
& \\
& \\
& \\
&
\end{aligned}
$$

with

$$
\begin{aligned}
& W_{20}(\theta)=\frac{i g_{20} \rho(0)}{\tau_{0} \omega_{0}} e^{i \tau_{0} \omega_{0} \theta}+\frac{i \bar{g}_{02} \bar{\rho}(0)}{3 \tau_{0} \omega_{0}} e^{-i \tau_{0} \omega_{0} \theta}+E_{1} e^{2 i \tau_{0} \omega_{0} \theta} \\
& W_{11}(\theta)=-\frac{i g_{11} \rho(0)}{\tau_{0} \omega_{0}} e^{i \tau_{0} \omega_{0} \theta}+\frac{i \bar{g}_{11} \bar{\rho}(0)}{\tau_{0} \omega_{0}} e^{-i \tau_{0} \omega_{0} \theta}+E_{2}
\end{aligned}
$$


where $E_{1}$ and $E_{2}$ can be obtained by the following two equations:

$$
\begin{aligned}
E_{1} & =\left(\begin{array}{ccccc}
2 i \omega_{0}-a_{11} & 0 & -a_{13} & 0 & -a_{15} \\
-a_{21} & 2 i \omega_{0}-a_{22} & -a_{23} & 0 & 0 \\
0 & -a_{32} & 2 i \omega_{0}-a_{33}-b_{33} e^{-2 i \tau_{0} \omega_{0}} & 0 & 0 \\
0 & 0 & -b_{43} e^{-2 i \tau_{0} \omega_{0}} & 2 i \omega_{0}-a_{44} & 0 \\
-a_{51} & 0 & 0 & 0 & 2 i \omega_{0}-a_{55}
\end{array}\right)^{-1} \\
& \times\left(\begin{array}{c}
E_{1}^{(1)} \\
E_{1}^{(2)} \\
E_{1}^{(3)} \\
E_{1}^{(4)} \\
0
\end{array}\right), \\
E_{2} & -\left(\begin{array}{ccccc}
a_{11} & 0 & a_{13} & 0 & a_{15} \\
a_{21} & a_{22} & a_{23} & 0 & 0 \\
0 & a_{32} & a_{33}+b_{33} & 0 & 0 \\
0 & 0 & b_{43} & a_{44} & 0 \\
a_{51} & 0 & 0 & 0 & a_{55}
\end{array}\right)^{-1} \times\left(\begin{array}{c}
E_{2}^{(1)} \\
E_{2}^{(2)} \\
E_{2}^{(3)} \\
E_{2}^{(4)} \\
0
\end{array}\right),
\end{aligned}
$$

with

$$
\begin{aligned}
& E_{1}^{(1)}=a_{16}+a_{17} \rho_{3}^{2}+a_{18} \rho_{3}, \\
& E_{1}^{(2)}=a_{24}+a_{25} \rho_{3}^{2}+a_{26} \rho_{3}, \\
& E_{1}^{(3)}=a_{34} \rho_{3}^{2} e^{-2 i \tau_{0} \omega_{0}}, \quad E_{1}^{(4)}=a_{45} \rho_{3}^{2} e^{-2 i \tau_{0} \omega_{0}}, \\
& E_{2}^{(1)}=2 a_{16}+2 a_{17} \rho_{3} \bar{\rho}_{3}+2 a_{18} \operatorname{Re}\left\{\rho_{3}\right\} \\
& E_{2}^{(2)}=2 a_{24}+2 a_{25} \rho_{3} \bar{\rho}_{3}+2 a_{26} \operatorname{Re}\left\{\rho_{3}\right\} \\
& E_{2}^{(3)}=2 a_{34} \rho_{3} \bar{\rho}_{3}, \quad E_{2}^{(4)}=2 a_{45} \rho_{3} \bar{\rho}_{3} .
\end{aligned}
$$

Then we can obtain

$$
\begin{aligned}
& C_{1}(0)=\frac{i}{2 \tau_{0} \omega_{0}}\left(g_{11} g_{20}-2\left|g_{11}\right|^{2}-\frac{\left|g_{02}\right|^{2}}{3}\right)+\frac{g_{21}}{2}, \\
& \mu_{2}=-\frac{\operatorname{Re}\left\{C_{1}(0)\right\}}{\operatorname{Re}\left\{\lambda^{\prime}\left(\tau_{0}\right)\right\}} \\
& \beta_{2}=2 \operatorname{Re}\left\{C_{1}(0)\right\} \\
& T_{2}=-\frac{\operatorname{Im}\left\{C_{1}(0)\right\}+\mu_{2} \operatorname{Im}\left\{\lambda^{\prime}\left(\tau_{0}\right)\right\}}{\tau_{0} \omega_{0}} .
\end{aligned}
$$

Thus, based on the properties of the Hopf bifurcation discussed in [24], we can get the following:

Theorem 2 The sign of $\mu_{2}$ determines the direction of the Hopf bifurcation: if $\mu_{2}>0$ $\left(\mu_{2}<0\right)$, then the Hopfbifurcation is supercritical (subcritical); the sign of $\beta_{2}$ determines the stability of the bifurcated periodic solutions: if $\beta_{2}<0\left(\beta_{2}>0\right)$, then the bifurcated periodic 
solutions are stable (unstable); and the sign of $T_{2}$ determines the period of the bifurcated periodic solutions: if $T_{2}>0\left(T_{2}<0\right)$, then the period of the bifurcated periodic solutions increases (decreases).

\section{Numerical simulation}

In this section, we try to present some numerical simulations for system (2) to validate the previous main results. By extracting some values from [22] and considering the conditions for the existence of the Hopf bifurcation, we choose a set of parameters as follows: $A=2$, $\delta_{0}=0.02, \alpha=0.27, \beta=0.003, c=0.01, \eta=0.2, \mu=0.003, \delta_{1}=0.2, \delta_{2}=0.045, \delta_{3}=0.03$, $a=0.4$. Then, we obtain the following specific case of system (2):

$$
\left\{\begin{array}{l}
\frac{d S(t)}{d t}=2-0.02 S(t)-\frac{0.27 S(t) I(t)}{S(t)+I(t)+0.01}+0.2 V(t)-0.003 S(t) \\
\frac{d E(t)}{d t}=\frac{0.27 S(t) I(t)}{S(t)+I(t)+0.01}-0.22 E(t) \\
\frac{d I(t)}{d t}=0.2 E(t)-0.05 I(t)-0.045 I(t-\tau)-\frac{0.003 I(t-\tau)}{I(t-\tau)+0.4} \\
\frac{d R(t)}{d t}=0.045 I(t-\tau)-0.02 R(t)+\frac{0.003 I(t-\tau)}{I(t-\tau)+0.4} \\
\frac{d V(t)}{d t}=0.003 S(t)-0.22 V(t)
\end{array}\right.
$$

Then Eq. (3) becomes

$$
-1.7084 e-004 I^{3}+0.0028 I^{2}+0.0023 I+4.6141 e-004=0 .
$$

By means of Matlab software package we can get the unique positive root $I_{*}=17.1823$ of Eq. (20). Then we get $\left[\alpha \delta_{1}-\left(\delta_{0}+\delta_{1}\right)\left(\delta_{0}+\delta_{2}+\delta_{3}\right)\right]\left(I_{*}+a\right)=0.5820>\beta \delta_{1}\left(\delta_{0}+\delta_{1}\right)=1.3200 e-$ 004. Thus we obtain the unique viral equilibrium $P_{*}(10.8619,8.3082,17.1832,38.8068$, 0.1481) of system (19).

By computation we obtain $\omega_{0}=3.5844, \tau_{0}=81.3618$, and $\lambda^{\prime}\left(\tau_{0}\right)=0.0041-0.0872 i$. As is shown in Figs. $1-3$, the viral equilibrium $P_{*}(10.8619,8.3082,17.1832,38.8068,0.1481)$ is locally asymptotically stable when $\tau=63.65<\tau_{0}=81.3618$. However, the viral equilibrium

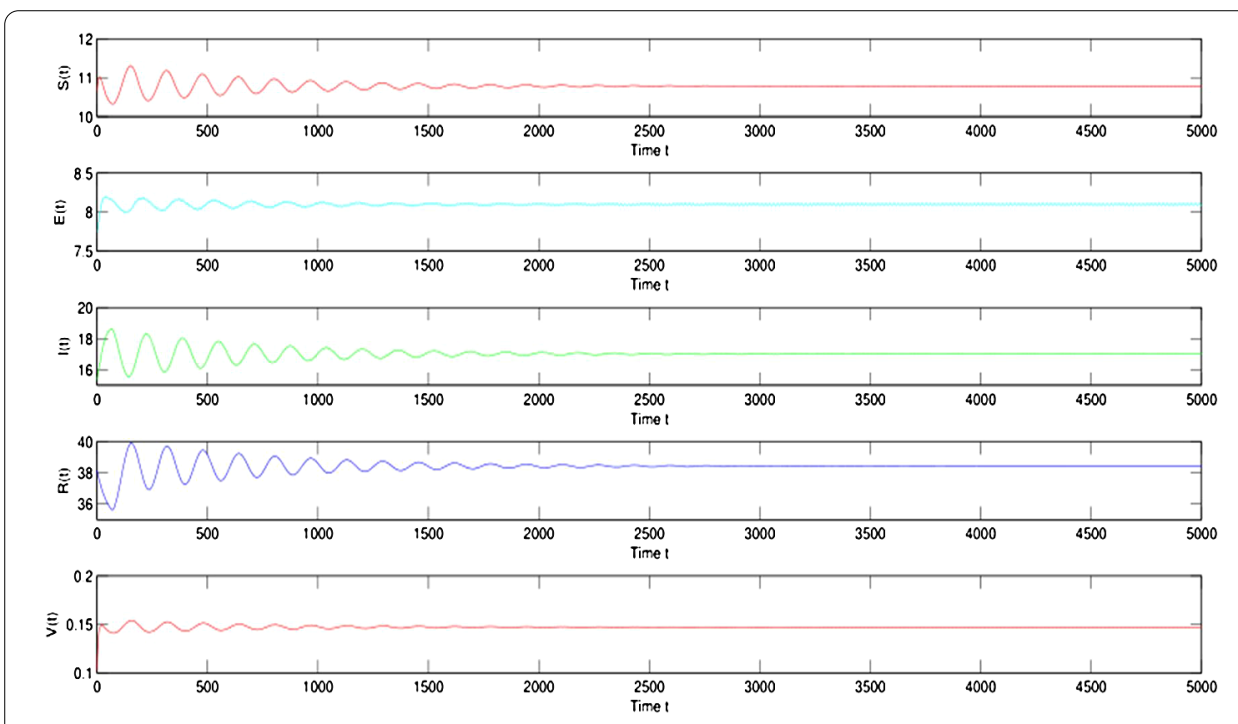

Figure 1 The trajectories of $S, E, I, R$, and $V$ with $\tau=63.65<\tau_{0}$ 


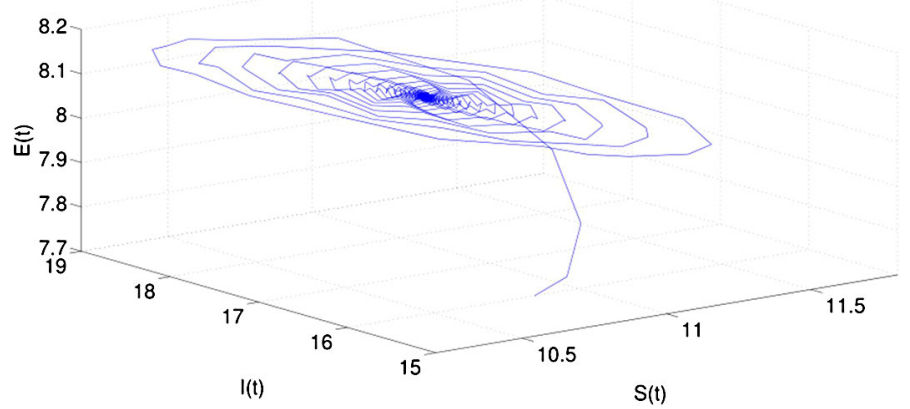

Figure 2 Dynamic behavior of system (19): projection on S-E-I with $\tau=63.65<\tau_{0}$

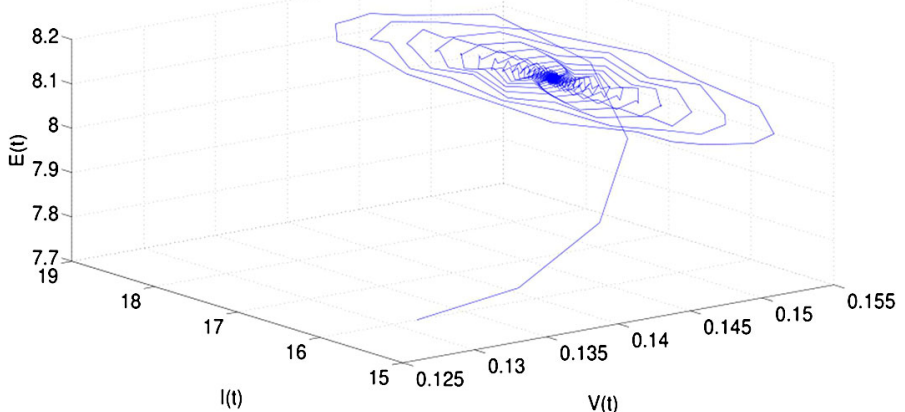

Figure 3 Dynamic behavior of system (19): projection on E-I-V with $\tau=63.65<\tau_{0}$

$P_{*}(10.8619,8.3082,17.1832,38.8068,0.1481)$ loses its stability, and a Hopf bifurcation occurs once $\tau>\tau_{0}=81.3618$, which can be exhibited by Figs. $4-6$ with $\tau=106.79$. This is consistent with the results in Theorem 1 . Therefore we can conclude that the propagation of the viruses in system (19) can be controlled by shortening the period that antivirus software uses to clean the viruses.

In addition, by some complex computations based on Eq. (18) we obtain $g_{20}=-3.7011+$ $6.8056 i, g_{11}=2.9207+0.6036 i, g_{02}=-3.7011-6.8056 i, g_{21}=-8.7200-3.3956 i$, and $C_{1}(0)=-4.4504-1.6676 i$. Further, we obtain $\beta_{2}=-8.9008<0, \mu_{2}=1085.5>0$, and $T_{2}=0.3303>0$. According to Theorem 2, the Hopf bifurcation is supercritical, the bifurcated periodic solutions are stable, and the period of the bifurcated periodic solutions increases. Therefore, the time delay due to the period that antivirus software uses to clean the viruses is harmful since the periodic behavior is unpleasant from the viewpoint of epidemiology. In practice, the stability of the computer virus system must be guaranteed to predict and even eliminate the viruses.

\section{Conclusions}

In this paper, we propose a delayed SVEIR computer virus model with nonlinear incident rate and saturated treatment rate by incorporating the time delay due to the period that antivirus software uses to clean the viruses in the infectious computers into the model considered in the literature [22]. Compared with the work in [22], the model considered 

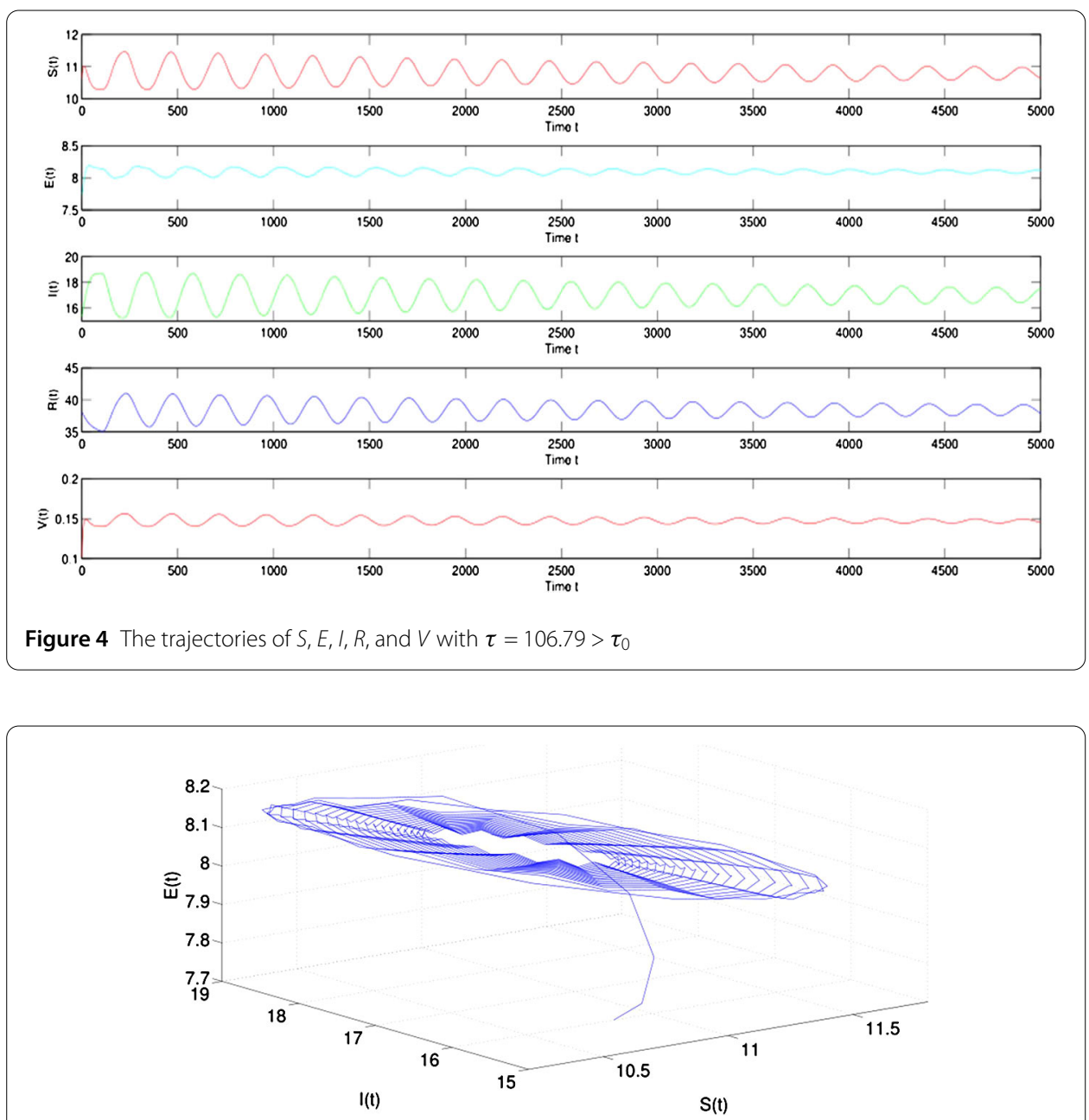

Figure 5 Dynamic behavior of system (19): projection on S-E-I with $\tau=106.79>\tau_{0}$

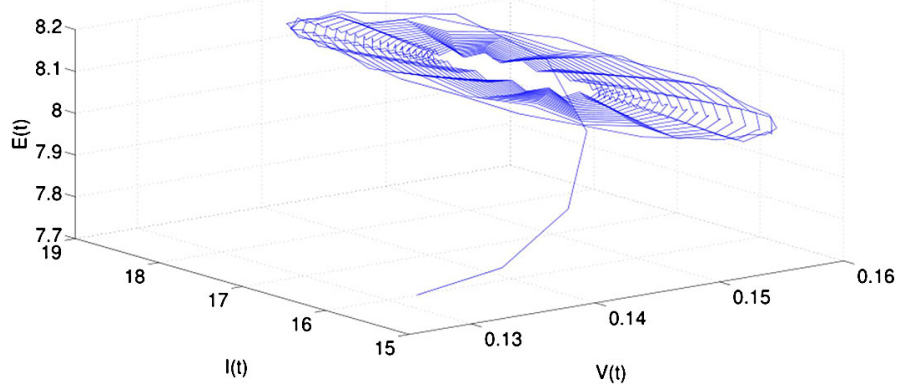

Figure 6 Dynamic behavior of system (19): projection on E-I-V with $\tau=106.79>\tau_{0}$

in the present paper is more general, and we mainly investigate the effects of the delay on the model.

The main results are given in terms of the stability of the viral equilibrium and Hopf bifurcation. We prove that the propagation of the viruses can be controlled when the value of the delay is below the critical value $\tau_{0}$. However, a Hopf bifurcation occurs when the 
value of the delay passes through the critical value $\tau_{0}$, which indicates that computers of the five classes in the model may coexist in an oscillatory mode under some conditions and the viruses will be out of control in this case. Therefore, we should control the occurrence of the Hopf bifurcation by using some bifurcation control strategies, and this will be a major emphasis of our future research.

\section{Acknowledgements}

The authors are grateful to the editor and the anonymous referees for their valuable comments and suggestions on the paper. This research was supported by Natural Science Foundation of Anhui Province (Nos. 1608085QF145, 1608085QF151).

\section{Funding}

This work was supported by Anhui Provincial Natural Science Foundation (Nos. 1608085QF145, 1608085QF151) and Project of Support Program for Excellent Youth Talent in Colleges and Universities of Anhui Province (No. gxyqZD2018044), without which this research would not be possible.

\section{Competing interests}

The authors declare that they have no competing interests.

\section{Authors' contributions}

The authors read and approved the final manuscript.

\section{Author details}

${ }^{1}$ School of Management Science and Engineering, Anhui University of Finance and Economics, Bengbu, China.

${ }^{2}$ Department of Applied Mathematics, Indian Institute of Technology (Indian School of Mines), Dhanbad, India.

\section{Publisher's Note}

Springer Nature remains neutral with regard to jurisdictional claims in published maps and institutional affiliations.

Received: 22 April 2018 Accepted: 4 July 2018 Published online: 27 July 2018

\section{References}

1. Chen, L.J., Sun, J.T.: Global stability and optimal control of an SIRS epidemic model on heterogeneous networks. Physica A 410, 196-204 (2014)

2. Han, X., Tan, Q.L.: Dynamical behavior of computer virus on Internet. Appl. Comput. Math. 217, 2520-2526 (2010)

3. Muroya, Y., Enatsu, Y., Li, H.X.: Global stability of a delayed SIRS computer virus propagation model. Int. J. Comput. Math. 91, 347-367 (2014)

4. Feng, L., Liao, X., Li, H., Han, Q.: Hopf bifurcation analysis of a delayed viral infection model in computer networks. Math. Comput. Model. 56, 167-179 (2012)

5. Ren, J.G., Yang, X.F., Yang, L.X., Xu, Y.H., Yang, F.Z.: A delayed computer virus propagation model and its dynamics. Chaos Solitons Fractals 45, 74-79 (2012)

6. Chen, J.Y., Yang, X.F., Gan, C.Q.: Propagation of computer virus under the influence of external computers: a dynamical model. J. Inf. Comput. Sci. 10, 5275-5282 (2013)

7. Gan, C.Q., Yang, X.F., Zhu, Q.Y., Jin, J., He, L.: The spread of computer virus under the effect of external computers. Nonlinear Dyn. 73, 1615-1620 (2013)

8. Yuan, H., Chen, G.: Network virus-epidemic model with the point-to-group information propagation. Appl. Comput. Math. 206, 357-367 (2008)

9. Dong, T., Liao, X., Li, H.: Stability and Hopf bifurcation in a computer virus model with multistate antivirus. Abstr. Appl. Anal. 2012, Article ID 841987 (2012)

10. Peng, M., He, X., Huang, J.J., Dong, T.: Modeling computer virus and its dynamics. Math. Probl. Eng. 2013, Article ID $842614(2013)$

11. Wang, F., Yang, F., Zhang, Y., Ma, J.: Stability analysis of a SEIQRS model with graded infection rates for Internet worms. J. Comput. 9, 2420-2427 (2014)

12. Liu, J.: Hopf bifurcation in a delayed SEIQRS model for the transmission of malicious objects in computer network. J. Appl. Math. 2014, Article ID 492198 (2014)

13. Mishra, B.K., Jha, N.: SEIQRS model for the transmission of malicious objects in computer network. Appl. Math. Model. 34, 710-715 (2010)

14. Yang, L.X., Yang, X., Wen, L., Liu, J.: A novel computer virus propagation model and its dynamics. Int. J. Comput. Math. 89, 2307-2314 (2012)

15. Yang, L.X., Yang, X., Liu, J., Zhu, Q., Gan, C.: Epidemics of computer viruses: a complex-network approach. Appl. Math. Comput. 219, 8705-8717 (2013)

16. Yang, X., Yang, L.X.: Towards the epidemiological modeling of computer viruses. Discrete Dyn. Nat. Soc. 2012, Article ID $259671(2012)$

17. Yao, Y., Xie, X.W., Guo, H., Yu, G., Gao, F.X., Tong, X.J.: Hopf bifurcation in an Internet worm propagation model with time delay in quarantine. Math. Comput. Model. 57, 2635-2646 (2013)

18. Amador, J.: The stochastic SIRA model for computer viruses. Appl. Math. Comput. 232, 1112-1124 (2014) 
19. Gan, C.Q., Yang, X.F., Zhu, Q.Y.: Propagation of computer virus under the influences of infected external computers and removable storage media. Nonlinear Dyn. 78, 1349-1356 (2014)

20. Wang, F., Zhang, Y., Wang, C., Ma, J.: Stability analysis of an e-SEIAR model with point-to-group worm propagation Commun. Nonlinear Sci. Numer. Simul. 20, 897-904 (2015)

21. Yang, L.X., Yang, X.F.: The impact of nonlinear infection rate on the spread of computer virus. Nonlinear Dyn. 82, 1-11 (2015)

22. Upadhyay, R.K., Kumari, S., Misra, A.K.: Modeling the virus dynamics in computer network with SVEIR model and nonlinear incident rate. J. Appl. Math. Comput. 54, 485-509 (2017)

23. Zhang, T.L., Jiang, H.J., Teng, Z.D.: On the distribution of the roots of a fifth degree exponential polynomial with application to a delayed neural network model. Neurocomputing 72, 1098-1104 (2009)

24. Hassard, B.D., Kazarinoff, N.D., Wan, Y.H.: Theory and Applications of Hopf Bifurcation. Cambridge University Press, Cambridge (1981)

25. Meng, X.Y., Huo, H.F., Zhang, X.B., Xiang, H.: Stability and Hopf bifurcation in a three-species system with feedback delays. Nonlinear Dyn. 64, 349-364 (2011)

26. Bianca, C., Ferrara, M., Gurrini, L.: The Cai model with time delay: existence of periodic solutions and asymptotic analysis. Appl. Math. Inf. Sci. 7, 21-27 (2013)

27. Gori, L., Gurrini, L., Sodini, M.: Hopf bifurcation in a Cobweb model with discrete time delays. Discrete Dyn. Nat. Soc. 2014, Article ID 137090 (2014)

\section{Submit your manuscript to a SpringerOpen ${ }^{\circ}$ journal and benefit from:}

- Convenient online submission

- Rigorous peer review

- Open access: articles freely available online

- High visibility within the field

Retaining the copyright to your article 